\title{
Review of: "A Fatal Case of Streptococcal Toxic Shock Syndrome Caused By Genotype Emm1 In The Third Trimester of Pregnancy, China"
}

Regev Cohen

Potential competing interests: The author(s) declared that no potential competing interests exist.

Lou et al described a fatal case of invasive GAS infection occurring in a young pregnant woman. I have several comments.

The case should be presented in more details, for example, did she underwent mechanical ventilation? Did she received noradrenaline? Was she transferred to the ICU? Did she have liver enzymes elevation? Did she have a typical rash? What was in the chest Xray? What were the chemistry and coagulation tests result? CRP? Which organs failed? The antibiotics that were given used should also appear in the text and not only in the figure, and the reasons for choosing them, should be discussed as well. Why clindamycin was not given empirically, at least until the results of the culture appeared? Was IVIG not available or the time frame did not allow for it to be administered?

Was the uterus cultured? Was it sent to pathological examination? Was the uterus necrotic? How do the authors explain the negative vaginal cultures?

Do the authors think that this woman had also influenzae? Was a PCR test for influenzae taken? What is the significance of testing IgM for this virus? Do the authors presume GAS was a complication of influenzae? Regarding the identification of GAS - aside from MS, was there identification using some automated machine? How was the susceptibility tested?

Peri-puerperal invasive GAS infection (involving the uterus) occur post or during labor. This case is unique in this aspect, appearing during the last trimester, prior to labor, and possibly not involving the uterus. GAS was cultured from the blood and sputum - did this woman suffered from GAS pneumonia, maybe after influenzae infection? If so, and if chest $X$ ray is supportive, it should be discussed and addressed in the Discussion.

What were the criteria that were fulfilled for the diagnosis of STSS? please elaborate.

The Discussion section is too general and do not add much to the manuscript at its present form. The first paragraph describe the fulminant nature of iGAS infection, which is known, although the rapid deterioration (within hours) in this case report is somewhat unique. The second paragraph deals with the difficulties in timely diagnosis of iGAS infection, and could be improved if more details are known to the reader, such as those described above (for example if the initial blood tests were compatible with viral infection, CRP was low etc.)

Another comment regarding Fig 2 . The $\mathrm{Y}$ axis probably represent the PIt counts, but surely not the PCT and 
$\mathrm{HgB}$. This should be corrected, or better, put in a table with the blood test results during the course of time.

The manuscript needs extensive English editing. 